\title{
Non-Surgical Management of Bile Leakage After Hepatectomy: A Single-Center Study
}

\author{
Takayoshi Kimura,* Tsuyoshi Kawai,†Yasufumi Ohuchi,* Shinsaku Yata,* Akira Adachi,* Youhei Takeda, \\ Kazuo Yashima, $\neq$ Soichiro Honjo, $§$ Naruo Tokuyasu $§$ and Toshihide Ogawa* \\ *Division of Radiology, Department of Pathophysiological and Therapeutic Science, School of Medicine, Tottori University Faculty of \\ Medicine, Yonago 683-8503, Japan, †Division of Radiology, Tottori Prefectural Kousei Hospital, Kurayoshi 682-0804, Japan, $¥$ Division \\ of Medicine and Clinical Science, Department of Multidisciplinary Internal Medicine, School of Medicine, Tottori University Faculty of \\ Medicine, Yonago 683-8504, Japan and \$Division of Surgical Oncology, Department of Surgery, School of Medicine, Tottori University \\ Faculty of Medicine, Yonago 683-8504, Japan
}

\section{ABSTRACT}

Background Bile leakage after hepatectomy is a common complication. The purpose of the present study was to retrospectively evaluate the usefulness of non-surgical management of bile leakage after hepatectomy, using 12year data from a single center study.

Methods Data from 15 patients (13 men, two women; mean age $67.1 \pm 7.0$ years) who had undergone nonsurgical management for bile leakage between January 2005 and November 2017 were retrospectively reviewed. Results We categorized bile leakage as central $(n=5)$ or peripheral $(n=10)$ leakage based on communication with the biliary tree. Percutaneous bile leakage drainage and/or endoscopic naso-biliary drainage (ENBD) $(n=2)$ or the rendezvous technique $(n=3)$ was successfully performed in five central-type cases, while all peripheral-type cases were treated with drainage alone; only one case required additional ethanol ablation. Bacterial bile cultures were positive in 11 cases and negative in four cases. The drainage catheters were removed after complete resolution in 13 cases $(86.7 \%)$, while two patients with cases of peripheral-type leakage died due to cancer progression while the drain was in place. No case needed conversion to reoperation. The mean duration of drainage therapy in all cases was $210.1 \pm 163.0$ days (range 17-531 days), with $316.8 \pm 180.8$ days in the central type and $156.7 \pm 131.5$ days in the peripheral type; this duration was not significantly different $(P=0.129)$.

Conclusion Non-surgical treatment is a minimally invasive and effective management strategy for postoperative bile leakage and the modality used depends on the type of bile leakage encountered.

Key words bile leakage; endoscopic naso-biliary drainage; percutaneous bile leakage drainage; percutaneous transhepatic biliary drainage; rendezvous technique

Bile leakage after hepatectomy is a common complication with an incidence rate of $2.9 \%-17 \% .^{1-9}$ The causes of bile leakage include cholerrhagia from the transected edge of the liver and sutural leakage after biliary tract rebuilding, among others. Bile leakage is also associated with serious biliary infection, and the resulting sepsis and septic shock are associated with high morbidity and mortality, impaired quality of life, and a substantial financial burden on both patients and society. ${ }^{2,10}$ Some previous literatures had reported some interactive cases as much volume of bilious discharge or major injury of biliary tree required repeat operation. However, the reoperation was considered to be risky and invasive extremely. Furthermore, surgical therapy for bile leakage in the postoperative period increases patient morbidity and mortality. ${ }^{11,}{ }^{12}$ Recently, treatment strategies for bile leakage have undergone fundamental changes due to the excellent results achieved using non-surgical management and minimally invasive interventions., 2, 5 Thus, currently, non-surgical treatment is regarded as the first choice in the management of postoperative bile leakage in a majority of cases, and these include modalities such as percutaneous bile leakage drainage (PBLD), biliary fistula ablation, endoscopic naso-biliary drainage (ENBD), or percutaneous transhepatic biliary drainage (PTBD). Sakamoto et al had reported excellent result of non-surgical treatment for postoperative bile-leakage cases, which was divided into two categories as with and without communication with the biliary tree. However, some of interactive cases needed surgical therapy in the postoperative period. ${ }^{1}$

Thus, the purpose of this study was to retrospectively evaluate the usefulness of non-surgical management of bile leakage after hepatectomy divided into two categories, based on data from 15 patients treated over a period of 12 years at our hospital.

\footnotetext{
Corresponding author: Takayoshi Kimura, MD

srkbc189@yahoo.co.jp

Received 2018 May 18

Accepted 2018 November 2

Abbreviations: CT, computed tomography; ENBD, endoscopic nasobiliary drainage; ERC, endoscopic retrograde cholangiography; $\mathrm{HCC}$, hepatocellular carcinoma; PBLD, percutaneous bile leakage drainage; PTBD, percutaneous transhepatic biliary drainage
} 


\section{MATERIALS AND METHODS Ethics}

This study was approved by the ethics committee of Tottori University (Approval No. 1BA139).

\section{Patients}

Patient characteristics are summarized in Table 1. Fifteen consecutive patients (13 men, two women; mean age $67.1 \pm 7.0$ years; range, 52 to 80 years) with bile leakage after open hepatectomy without biliary reconstruction were treated using non-surgical methods between January 2005 and November 2017 at our hospital and their charts were reviewed retrospectively. Of the 15 patients, seven $(46.7 \%)$ had hepatocellular carcinoma (HCC), seven (46.7\%) had liver metastases, and one case $(6.7 \%)$ involved a hilar cholangiocarcinoma. Liver metastases were from gastric cancer in two cases (13.3\%), colon cancer in four cases (26.7\%), and an undifferentiated high grade pleomorphic sarcoma in one case (6.7\%). A partial hepatectomy or segmentectomy (segments 2, 3, 4,7 , or 8 ) had been performed in five cases (33.3\%), an anterior sectionectomy in four cases $(26.7 \%)$, a posterior sectionectomy in two cases (13.3\%), an extended hepatectomy or left hepatectomy in two cases (13.3\%), a right hepatectomy in one case (6.7\%), and a central bisectionectomy in one case $(6.7 \%)$. The sites of bile leakage were at the transected edge of the liver in all cases.

\section{Procedure}

Our strategy for non-surgical treatment of bile leakage is shown in Fig. 1. Abdominal drains that had been placed during surgery were removed a few days post procedure, when the drain fluid was grossly serous and had gradually decreased or disappeared. If bile leakage was suspected based on symptoms such as high fever or jaundice, a computed tomography (CT) scan was performed to detect abdominal fluid collection. If bile leakage was indeed detected, PBLD using an $8.5 \mathrm{Fr}$ drainage catheter (Dawson-Mueller Multipurpose Drainage Catheter, Cook Medical, Bloomington, IN) and/or endoscopic retrograde cholangiography (ERC) was performed under CT guidance. In this study, we classified post-operative bile leakage into two categories, according to Sakamoto's classification. ${ }^{1}$ If the leaky bile ducts were confirmed to be in communication with the biliary tree, either by fistulography via the PBLD catheter or by endoscopic retrograde cholangiography (ERC), such leakage was defined as "central-type," whereas leaky bile ducts that were not in communication with the biliary tree were defined as "peripheral-type." Appropriate antibiotic therapy was administered based on bacterial cultures of the drainage fluid.
Table 1. Baseline Characteristics of 15 cases

\begin{tabular}{ll}
\hline Parameter & $\begin{array}{l}\text { Value, } \\
\text { No. of patients }\end{array}$ \\
\hline $\begin{array}{l}\text { Age in years } \\
\text { Gender }\end{array}$ & $67.1 \pm 7.0(52-80)$ \\
Male & $13(86.7 \%)$ \\
Female & $2(13.3 \%)$ \\
Disease & $7(46.7 \%)$, \\
HCC & $7(46.7 \%)$ \\
Liver metastases & $2(13.3 \%)$ \\
$\quad$ Gastric cancer & $4(26.7 \%)$ \\
$\quad$ Colon cancer & $1(6.7 \%)$ \\
$\quad \begin{array}{l}\text { Undifferentiated high grade pleomor- } \\
\text { phic sarcoma }\end{array}$ & $1(6.7 \%)$ \\
Hilar cholangiocarcinoma & \\
Type of hepatectomy & $5(33.3 \%)$ \\
Partial hepatectomy or segmentectomy & \\
(2, 3, 4, 7, 8) & $2(13.3 \%)$ \\
(Extended) left hepatectomy & $4(26.7 \%)$ \\
Anterior sectionectomy & $2(13.3 \%)$ \\
Posterior sectionectomy & $1(6.7 \%)$ \\
Right hepatectomy & $1(6.7 \%)$ \\
Central bisectionectomy & $15(100 \%)$ \\
\hline Sites of bile leakage & \\
\hline Transection edge of liver & \\
\hline
\end{tabular}

Values represent mean \pm SD (range) or number (\%). HCC, hepatocellular carcinoma.

In patients with central-type leakage, ENBD was performed as an initial biliary intervention using 5.0 to $6.0 \mathrm{Fr}$ drainage catheter (Flexima, Boston Scientific, Watertown, MA) to divert bile flow away from the leakage site and reduce bile duct pressure. When bilious discharge from the drainage catheter was greater than $100 \mathrm{~mL}$ per day and persisted for several weeks after the initial diverting procedure or if the catheter could not be placed across the bile leakage site, PTBD using an 8.5Fr drainage catheter (Dawson-Mueller Multipurpose Drainage Catheter, Cook Medical, Bloomington, IN) was performed to additionally decompress the biliary tree. If the drainage catheter could not be placed across the site of bile duct injury, the rendezvous technique was performed using two separate pathways, wherein both endoscopic and percutaneous bile duct access routes to reach one common site were created, with subsequent capture of the trailing end of one access wire with a snare catheter was positioned through the second access site. ${ }^{13-16}$

In patients with non-effective peripheral-type leakage, if bilious discharge greater than $100 \mathrm{~mL}$ per day from the drainage catheter persisted for several weeks, ethanol ablation therapy for a biliary fistula via PBLD tube was considered as the additional biliary intervention.

In patients with central- or peripheral-type leakage, 


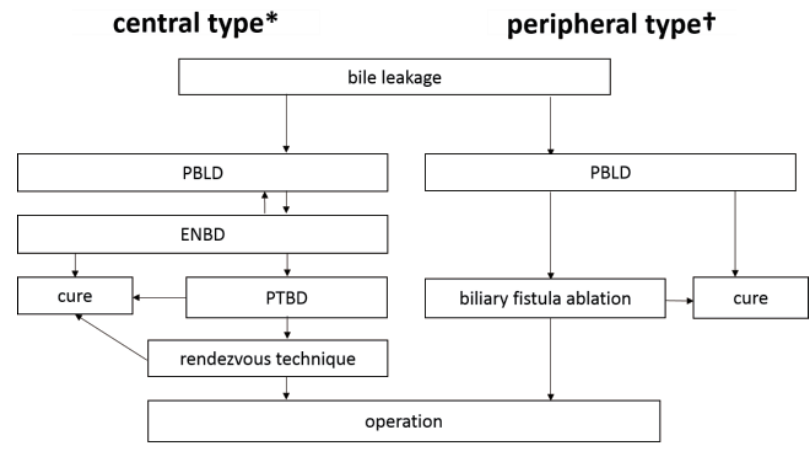

Fig. 1. Non-surgical treatment strategy for bile leakage.This figure illustrates our strategy of non-surgical treatment for bile leakage. *Central type: in communication with a biliary tree; †peripheral type: not in communication with a biliary tree. ENBD, endoscopic naso-biliary drainage; PBLD, percutaneous bile leakage drainage; PTBD, percutaneous transhepatic biliary drainage.

a fistulography and/or cholangiography was regularly performed once every one or two weeks to confirm resolution of bile leakage. A reoperation was considered only if these treatments failed.

The drain was clamped when bilious discharge from drainage catheter had ceased, if bile leakage had reduced significantly, or if it had almost disappeared when evaluated in a CT scan. Finally, the drain was removed only when there was no recurrence of symptoms and no elevation of biochemical parameters such as a bilirubin, a biliary enzyme and an inflammatory response.

\section{Analysis}

The medical records and endoscopic and radiologic findings were reviewed retrospectively. Clinical parameters, including type of hepatectomy, central- or peripheral-type leakage, bacterial cultures, the necessity for additional biliary intervention, and mean duration of continuous drainage therapy were evaluated using the F-test and Student's $t$-test. $P$-values $<0.05$ were considered statistically significant. IBM SPSS statistics 22.0 software (IBM Corporation, Armonk, NY) was used for all statistical analyses.

\section{RESULTS}

The outcomes after non-surgical therapy for bile leakage are provided in Table 2 and Fig. 2. Also, the outcomes between the central-type and peripheral-type groups are provided in Table 3. Initial biliary intervention could be successfully performed in all 15 cases. Among the 15 patients with confirmed bile leakage, central-type and peripheral-type leakages were observed in five (33.3\%) and $10(66.7 \%)$ cases, respectively. In both central- and peripheral-type leakage, fistulography and/or cholan- giography was performed to confirm cessation of bile leakage every one or two weeks.

For all cases combined, the mean interval from operation to initial biliary intervention was $68.1 \pm 81.9$ days (13-267 days). The drainage catheter was removed after complete resolution and without recurrence of bile leakage in 13 cases $(86.7 \%)$ that were treated by non-surgical methods alone. The mean duration of drainage therapy for both groups combined was $210.1 \pm 163.0$ days (range 17-531 days). Both mean interval from operation to biliary intervention and mean duration of continuous drainage therapy were not significantly different between the central-type and peripheral-type groups $(P=0.897$ and $P=0.129$, respectively). None of the patients required reoperation.

In patients with central-type leakage, the mean interval from operation to initial biliary intervention was $64.8 \pm 49.7$ days (range 13-122 days). PBLD was performed prior to ENBD in three cases, and ENBD was performed prior to PBLD caused by occlusive jaundice in remaining two cases. Two of the five patients with central-type leakage were successfully managed using PBLD and ENBD, while in the other three cases PTBD was required as additional biliary intervention because of non-effective drainage or ENBD failure. Further, in all these three cases, the drainage catheter for PTBD could not be placed across the bile leakage site because of a large biliary fistula. Therefore, the rendezvous technique was performed on days 14, 14, and 160 after initial biliary intervention in these three patients, respectively. The median healing duration for all five cases of central-type leakage was 316.8 days (range 145-531 days).

In patients with peripheral-type leakage, the mean interval from operation to initial biliary intervention was $69.8 \pm 96.6$ days (16-267 days), and PBLD alone was sufficient for leakage resolution in seven of the 10 cases. The median period of healing was 156.7 days (range 17-411 days). Additional biliary intervention, in the form of ethanol ablation for biliary fistula via PBLD, was required only in one case and was performed 224 days after initial biliary intervention; the fistula healed after 355 days. Two cases with peripheral-type leakage died due to cancer progression during the median drainage period of 316.0 days (range 211-411 days).

Bacterial cultures from the drainage bile fluid were positive in 11 cases $(73.3 \%$ ) and negative in four cases (26.7\%); four positive cases of five central-type cases (80\%), and seven positive cases of ten peripheral-type cases $(70 \%)$, respectively. Of the 11 positive cases, bacteria were of intestinal origin in seven cases $(46.7 \%)$ and of dermal origin in four cases (26.7\%). The mean duration of continuous drainage therapy was slightly longer in the positive group than in the negative group but was not 
Table 2. Outcomes of non-surgical treatment for bile leakage (over all)

\begin{tabular}{lc}
\hline Parameter & Value, No. of patients $P$-values \\
\hline Technical success of initial biliary intervention & $15(100 \%)$ \\
Type of bile leakage & $5(33.3 \%)$ \\
$\quad$ Central-type & $10(66.7 \%)$. \\
$\quad$ Peripheral-type & $68.1 \pm 81.9(13-267)$ \\
Mean interval from operation to initial biliary intervention, days & \\
Additional biliary intervention & $4(26.7 \%)$ \\
$\quad$ Yes & $11(73.3 \%)$ \\
$\quad$ No (initial biliary intervention alone) & $210.1 \pm 163.0(17-531)$ \\
Mean duration of drainage therapy, days & $11(73.3 \%)$ \\
Bile culture & $7(46.7 \%)$ \\
$\quad$ Positive & $4(26.7 \%)$ \\
$\quad$ Bacteria derived from intestine & $4(26.7 \%)$. \\
$\quad$ Bacteria derived from skin & \\
$\quad$ Negative & $238.3 \pm 174.1(77-531)$ \\
$\quad$ Mean duration of drainage therapy, days & $P=0.197$ \\
$\quad$ Positive bile cultivation & $132.5 \pm 109.5(17-530)$ \\
Negative bile cultivation & $13(86.7 \%)$ \\
Clinical outcomes & $2(13.3 \%)$ \\
$\quad$ Successful removal of drainage catheter & $0(0 \%)$ \\
\hline Died with cancer progression remained drainage catheter & $0(0 \%)$ \\
\hline Reoperation & \\
\hline Vales reing continuous drainage therapy & \\
\hline
\end{tabular}

Values represent mean \pm SD (range) or number (\%).

Table 3. Outcomes of non-surgical treatment for bile leakage (central-type vs. peripheral-type)

\begin{tabular}{|c|c|c|c|c|c|}
\hline Parameter & Central-type $(n=5)$ & & Peripheral-type $(n=10)$ & & $P$-values \\
\hline $\begin{array}{l}\text { Mean interval from operation to initial biliary intervention, } \\
\text { days }\end{array}$ & $64.8 \pm 49.7(13-122)$ & & $69.8 \pm 96.6(16-267)$ & & $P=0.897$ \\
\hline Additional biliary intervention & Rendezvous technique & 3 & Ethanol ablation & 1 & \\
\hline Mean duration of drainage therapy, days & $316.8 \pm 180.8(145-531)$ & & $156.7 \pm 131.5(17-411)$ & & $P=0.129$ \\
\hline \multirow[t]{2}{*}{ Bile culture } & Positive & 4 & Positive & 7 & \\
\hline & Negative & 1 & Negative & 3 & \\
\hline Mean duration of drainage therapy with positive group, days & $338.3 \pm 201.4(145-531)$ & & $181.1 \pm 140.6(17-411)$ & & $P=0.226$ \\
\hline \multicolumn{6}{|l|}{ Clinical outcomes } \\
\hline Successful removal of drainage catheter & 5 & 8 & & & \\
\hline Died with cancer progression remained drainage catheter & 0 & 2 & & & \\
\hline Undergoing continuous drainage therapy & 0 & 0 & & & \\
\hline Reoperation & 0 & 0 & & & \\
\hline
\end{tabular}

Values represent mean $\pm \mathrm{SD}$ (range) or number (\%).

significantly different $(P=0.197)$. As limited to the positive group, the mean duration of continuous drainage therapy was slightly longer in the central-type than in peripheral-type but was not significantly different $(P=$ 0.226).

Figure 3 illustrates the rendezvous technique performed in a 60-year-old male with bile leakage after a central bisectionectomy of the liver for HCC. CT images at 65 days after surgery showed bile leakage at the transected edge of the liver. ENBD was initially performed on day 119 after the central bisectionectomy; however, the drainage catheter could not be advanced into the peripheral bile duct over the injury site. Therefore, subsequently, PBLD and PTBD via the lateral inferior subsegment branch (B3) were simultaneously performed under ultrasonography guidance on day 122 after surgery. However, the guide wire could not be advanced into the bile duct across the leakage site. Bacterial culture of the PBLD drainage fluid yielded intestinal bacteria. Bilious discharge of over $300 \mathrm{~mL}$ per day from the PBLD per- 


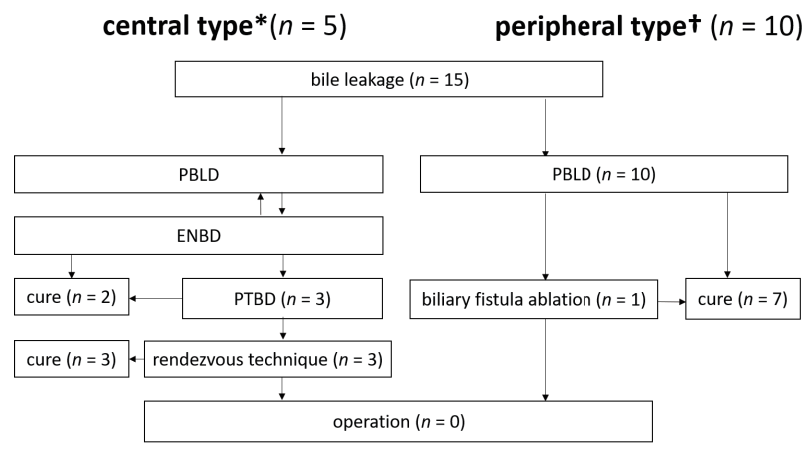

Fig. 2. Outcomes after non-surgical treatment for bile leakage. This figure shows the outcomes after non-surgical treatment for bile leakage in this study. None of the cases, either with central-type or peripheral-type, required continuous drainage therapy or conversion to reoperation. *Central type: in communication with a biliary tree, †peripheral type: not in communication with a biliary tree. ENBD, endoscopic naso-biliary drainage; PBLD, percutaneous bile leakage drainage; PTBD, percutaneous transhepatic biliary drainage.

sisted for eight weeks, and a percutaneous fistulogram via the drainage catheter demonstrated communication with the biliary tree, indicating the presence of a centraltype leakage. Therefore, the rendezvous technique was performed on day 136 after surgery, as follows. A loop-grasping snare was placed in the PTBD catheter and was used to grasp the endoscopic guide wire, which was threaded through the ENBD catheter. Subsequently, the PTBD catheter was successfully placed over the bile leakage site via the rendezvous procedure, bypassing the leakage site. A percutaneous fistulogram via the drainage catheter demonstrated persistent communication with the biliary tree. Bilious discharge via the PBLD decreased gradually and as CT showed the disappearance of bile leakage, drainage catheters were successfully removed on day 653 post surgery.

\section{DISCUSSION}

Bile leakage after hepatectomy is a common complication with an incidence of $2.9 \%-17 \%,{ }^{1-9}$ and currently non-surgical treatment is being regarded as first choice in the management of postoperative bile leakage. Effective therapy is selected based on the type of bile leakage, which is divided into two categories, as with (central-type) and without communication (peripheraltype) with the biliary tree. ${ }^{1}$ Central-type leakage is predominantly and effectively treated by PBLD and ENBD, which reduce internal pressure in the bile duct and decrease leakage volume. ${ }^{1-9}$ A combination of biliary intervention therapies, such as the rendezvous technique,

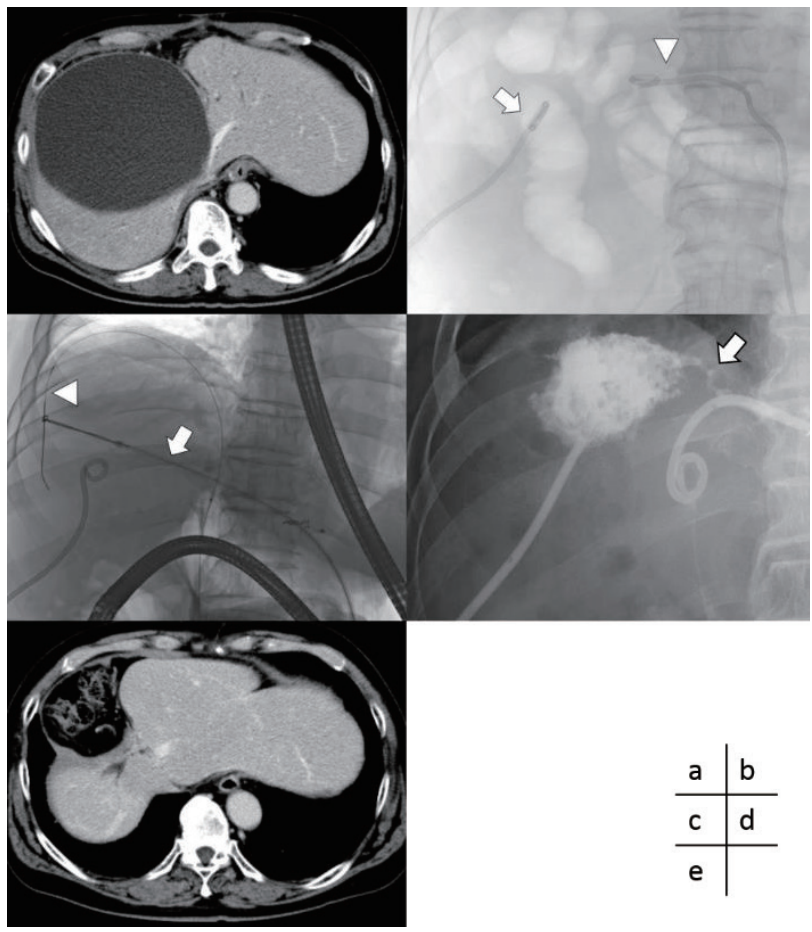

Fig. 3. A 60-year-old male presented with a bile leakage after central bisectionectomy of the liver for hepatocellular carcinoma. (a) $\mathrm{CT}$ at 65 days after surgery showed bile leakage at the transected edge of the liver after central bisectionectomy. (b) ENBD was performed 119 days after surgery; however, the drainage catheter could not be advanced into the peripheral bile duct over the injury site. Subsequently, PBLD (arrow) and PTBD (arrowhead) were simultaneously performed via the lateral inferior subsegment branch (B3) under US guidance on day 122 after surgery; however, the guide wire could not be advanced into the bile duct across the leakage site. (c) The rendezvous technique was performed 136 days after surgery, wherein a loop grasping snare (arrow) placed into the bile leakage via the PTBD catheter was used to grasp the endoscopic guide wire (arrowhead) that was threaded through the ENBD catheter. (d) The PTBD catheter was placed over the bile leakage site successfully using the rendezvous procedure, thus bypassing the leakage site. A percutaneous fistulogram via the drainage catheter demonstrated persistence of communication with the biliary tree (arrow). (e) CT showed disappearance of bile leakage, and drainage catheters were successfully removed on day 653 after surgery. CT, computed tomography; ENBD, endoscopic naso-biliary drainage; PBLD, percutaneous bile leakage drainage; PTBD, percutaneous transhepatic biliary drainage.

should also be considered as initial therapeutic options in cases that therapeutic effect of PBLD and ENBD is insufficient. In this study, the rendezvous technique was successfully performed after ENBD failure to reduce bile duct pressure in three of five central-type cases. All central-type leakage cases were resolved within a median period of 316.8 days (range 145-531 days). On the other hand, for peripheral type leakage, PBLD is first choice 
of treatment. Some intractable cases of peripheral-type leakage have been reported to require newer non-surgical interventional therapies, including biliary ablation with absolute ethanol, because ethanol affects the remaining bile duct and causes irreversible damage. In this study, all peripheral-type cases, except one, resolved after drainage alone and within a median period of 156.7 days (range 17-411 days). Only one case required ethanol ablation of a biliary fistula through PBLD after drainage. Importantly, in both types of leakage, surgical biliary reconstruction with strong invasion should only be considered when there are no essential conditions and if the non-surgical management strategies stated above prove ineffective. ${ }^{17-19}$ Further, mismanagement of the condition should be avoided in all respects. In this study, all cases of bile leakage could be resolved using non-surgical methods alone. Sakamoto et al. (2016) had reported some cases required the reoperation as the non-surgical treatments failed. ${ }^{1}$ In our study, none of the cases required repeat surgery, because our strategy of bile leakage could be more effective than previous reports. We perform PBLB, ENBD and PTBD for biliary tract decompression positively and also tried to be placed the drainage catheter across a biliary tract failure site by performing the rendezvous technique. The reoperation was considered to be risky and invasive extremely. Therefore, it is important that we were able to avoid much invasive repeat surgery by the combined various non-surgical treatments including PBLD, ENBD, PTBD and rendezvous technique, especially in central-type.

Sakamoto et al. (2016) have reported that patients who underwent segmentectomy 1 , medial sectionectomy, anterior sectionectomy, or central bisectionectomy were found to be at high risk for bile leakage. ${ }^{1}$ Nagano et al. (2003) have demonstrated that hepatectomies, wherein the cut surface exposes the major Glisson's sheath and includes the hepatic hilum, are independent risk factors for bile leakage. ${ }^{5}$ However, the correlation between the type of hepatectomy and postoperative bile leakage has not yet been clearly defined, as is the correlation with the occurrence of central- or peripheral-type leakage. As hepatectomy including one of the central bisections or the hepatic hilum showed a tendency to develop central-type leakage, further prospective studies of many cases are necessary because of only five central type cases in this study.

In our study, successful non-surgical management of bile leakage was achieved in all 15 patients; this corresponds to a cure rate of $86.7 \%$ as two patients died due to cancer progression with the drainage catheter remaining in place. Previous studies have reported cure rates that range from $82 \%-90 \%$, including endoscopic management. ${ }^{1,2,10,19-22}$ The cure rate of drainage therapy achieved in our facility is comparable to that of previous reports. The mean duration of drainage therapy in our study was $210.1 \pm 163.0$ days, which is similar to that of previous reports that ranged from two to 24 months., ${ }^{11}$, 18, 21-25 Further, we found that mean duration of drainage therapy in central-type leakage tends to be longer than that of peripheral-type leakage (316.8 days vs. 156.7 days, $P=0.129$ ). This observation, in combination with the fact that peripheral-type leakage is associated with lower leakage volume, probably accounts for why many cases of peripheral-type leakage can be resolved by PBLD alone. On the other hand, many cases of centraltype several additional biliary intervention including PTBD or rendezvous technique combined with initial PBLD or ENBD, because initial drainage therapy alone is not effective in resolving large leakage volumes. In our study, the central-type group required additional biliary intervention initially when the drainage volume was higher along with greater severity of the clinical course and protraction of the treatment period.

Cases testing positive for bacterial culture were found at almost about the same high frequency in both central-type and peripheral-type groups (80\% vs. $70 \%$ ). Cases testing positive for bacterial culture tended to have a longer duration of drainage therapy than the negative group but was not significantly different (238.3 days vs. 132.5 days, $P=0.197)$. As limited to the positive group, the mean duration of continuous drainage therapy was slightly longer in the central-type than in peripheral-type but was not significantly different $(P=0.226)$. Generally, bacterial bile infections are treated using antibiotics; however, it is often difficult to select appropriate susceptible antibiotics. Further, the general condition of the patient might worsen even if the proper antibiotics are administered, thus resulting in longer treatment periods. ${ }^{26}$ Therefore appropriate antibiotics should be used until cholangitis is improved clinically, and bacterial bile leakage can continue until complete recovery in the treatment period, regardless of the bacterial culture results. If multiple strategies have been used to resolve bile leakage, it is necessary to ensure proper placement of the drainage catheter, check for change in cavity size using CT, ERC, and PTC, and ascertain the appropriate removal period. Importantly, infection-induced ENBD and PTBD should also be considered as ENBD may promote a retrograde infection at the duodenal papilla and PTBD may increase bacterial infection derived from the skin.

When considered in its entirety, our results indicate that non-surgical therapy for bile leakage after hepatectomy is adequately efficacious and safe. Nonetheless, as the present study is retrospective, the optimal therapeutic strategy for 
bile leakage could not be identified. Larger prospective studies are needed to determine the optimal strategy.

In conclusion, non-surgical management can be a minimally invasive and effective approach for treating postoperative bile leakage, but strategy selection should be based on whether the leakage is the central-type or the peripheral-type. Specifically, as central-type leakage is refractory to therapy and require a long time for resolution, ENBD, PTBD or the rendezvous technique should be considered in central-type leakage to reduce bile duct pressure when placed across the bile leakage site.

Acknowledgments: The authors thank Tsuyoshi Kawai, Yasufumi Ohuchi and Toshihide Ogawa for their editorial assistance in preparing the manuscript.

The authors declare no conflict of interest.

\section{REFERENCES}

1 Sakamoto K, Tamesa T, Yukio T, Tokuhisa Y, Maeda Y, Oka M. Risk factors and managements of bile leakage after hepatectomy. World J Surg. 2016;40:182-9. PMID: 26159119.

2 Chen XP, Peng SY, Peng CH, Liu YB, Shi LB, Jiang XC, et al. A ten-year study on non-surgical treatment of postoperative bile leakage. World J Gastroenterol. 2002;8:937-42. PMID: 12378646.

3 Yamashita Y, Hamatsu T, Rikimaru T, Tanaka S, Shirabe K, Shimada M, et al. Bile leakage after hepatic resection. Ann Surg. 2001;233:45-50. PMID: 11141224.

4 Guillaud A, Pery C, Campillo B, Lourdais A, Sulpice L, Boudjema K. Incidence and predictive factors of clinically relevant bile leakage in the modern era of liver resections. HPB. 2013;15:224-9. PMID: 23374363.

5 Sadamori H, Yagi T, Shinoura S, Umeda Y, Yoshida R, Satoh $\mathrm{D}$, et al. Risk factors for major morbidity after liver resection for hepatocellular carcinoma. Br J Surg. 2013;100:122-9. PMID: 23175234.

6 Nagano Y, Togo S, Tanaka K, Masui H, Endo I, Sekido H, et al. Risk factors and management of bile leakage after hepatic resection. World J Surg. 2003;27:695-8. PMID: 12732991.

7 Reed DN Jr, Vitale GC, Wrightson WR, Edwards M, McMasters K. Decreasing mortality of bile leaks after elective hepatic surgery. Am J Surg. 2003;185:316-8. PMID: 12657381.

8 Tanaka S, Hirohashi K, Tanaka H, Shuto T, Lee SH, Kubo S, et al. Incidence and management of bile leakage after hepatic resection for malignant hepatic tumors. J Am Coll Surg. 2002;195:484-9. PMID: 12375753.

9 Terajima H, Ikai I, Hatano E, Uesugi T, Yamamoto Y, Shimahara Y, et al. Effectiveness of endoscopic nasobiliary drainage for postoperative bile leakage after hepatic resection. World J Surg. 2004;28:782-6. PMID: 15457358.

10 De Robertis R, Contro A, Zamboni G, Mansueto G. Totally percutaneous rendezvous techniques for the treatment of bile strictures and leakages. J Vasc Interv Radiol. 2014;25:650-4. PMID: 24674222.

11 Abdel-Raouf A, Hamdy E, El-Hanafy E, El-Ebidy G. Endoscopic management of postoperative bile duct injuries: a single center experience. Saudi J Gastroenterol. 2010;16:19-24. PMID: 20065569.

12 Costamagna G, Pandolfi M, Mutignani M, Spada C, Perri V. Long-term results of endoscopic management of postoperative bile duct strictures with increasing numbers of stents. Gastrointerest Endosc. 2001;54:162-8. PMID: 11474384.

13 Nasr JY, Hashash JG, Orons P, Marsh W, Slivka A. Rendezvous procedure for the treatment of bile leaks and injury following segmental hepatectomy. Dig Liver Dis. 2013;45:433-6. PMID: 23352315.

14 Saleem A, Leroy AJ, Baron TH. Modified rendezvous technique with successful reconnection of completely transected common bile duct using combined endoscopic and radiologic approach. Endoscopy. 2010;42:178-9. PMID: 20640979.

15 Aytekin C, Boyvat F, Yimaz U, Harman A, Haberal M. Use of the rendezvous technique in the treatment of biliary anastomotic disruption in a liver transplant recipient. Liver Transpl. 2006;12:1423-6. PMID: 16933230.

16 Miraglia R, Traina M, Maruzzelli L, Caruso S, Di Pisa M, Gruttadauria S, et al. Usefulness of the "rendezvous" technique in living related right liver donors with postoperative biliary leakage from bile duct anastomosis. Cardiovasc Intervent Radiol. 2008;31:999-1002. PMID: 18196331.

17 Suzuki D, Kato A, Kimura F, Shimizu H, Yoshidome H, Ohtsuka M, et al. Four case of biliary leakage after hepatobiliary-pancreatic surgery managed by biliary ablation with absolute ethanol. Tando. 2009;23:188-93. DOI: 10.11210/ tando.23.188.

18 Sadakari Y, Miyoshi A, Ohtsuka T, Kohya N, Takahashi T, Matsumoto K, et al. Percutaneous transhepatic portal embolization for persistent bile leakage after hepatic resection: report of a case. Surg Today. 2008;38:668-71. PMID: 18612797.

19 Yamakado K, Nakatsuka A, Iwata M, Kondo A, Isaji S, Uemoto S, et al. Refractory biliary leak from intrahepatic biliary-enteric anastomosis treated by selective portal vein embolization. J Vasc Interv Radiol. 2002;13:1279-81. PMID: 12471195.

20 Kim KH, Kim TN. Endoscopic management of bile leakage after cholecystectomy: a single-center experience for 12 years. Clin Endosc. 2014;47:248-53. PMID: 24944989.

21 Binmoeller KF, Katon RM, Shneidman R. Endoscopic management of postoperative biliary leaks: review of 77 cases and report of two cases with biloma formation. Am J Gastroenterol. 1991;86:227-31. PMID: 1992640.

22 Fujii T, Maguchi H, Obara T, Tanno S, Itoh A, Shudo R, et al. Efficacy of endoscopic diagnosis and treatment for postoperative biliary leak. Hepatogastroenterology. 1998;45:656-61. PMID: 9684112.

23 Uller W, Müller-Wille R, Loss M, Hammer S, Schleder S, Goessmann H, et al. Percutaneous management of postoperative bile leaks with an ethylene vinyl alcohol copolymer (Onyx). Rofo. 2013;185:1182-7. PMID: 23860801.

24 Stanpfl U, Hackert T, Radeleff B, Sommer CM, Stampfl S, Werner J, et al. Percutaneous management of postoperative bile leaks after upper gastrointestinal surgery. Cardiovasc Intervent Radiol. 2011;34:808-15. PMID: 21301846.

25 Civelli EM, Meroni R, Cozzi G, Milella M, Suman L, Vercelli $\mathrm{R}$, et al. The role of interventional radiology in biliary complications after orthotopic liver transplantation: a single-center experience. Eur Radiol. 2004;14:579-82. PMID: 14648104.

26 Shimizu J, Kaibori M, Murakami M, Noda T, Dono K, Matsui $\mathrm{K}$, et al. Multicenter study of post-hepatectomy bile leakage and bacterial infections. J Jpn Soc Surg Infect. 2013;10:725-8. 